\title{
Effectiveness of aquatic exercises compared to patient- education on health status in individuals with knee osteoarthritis: A randomized controlled trial.
}

\begin{tabular}{|c|c|}
\hline Journal: & Clinical Rehabilitation \\
\hline Manuscript ID & CRE-2017-6315.R1 \\
\hline Manuscript Type: & Original Article \\
\hline Keywords: & Self-Care, Hydrotherapy, Knee Osteoarthritis, pain, Depression \\
\hline Abstract: & 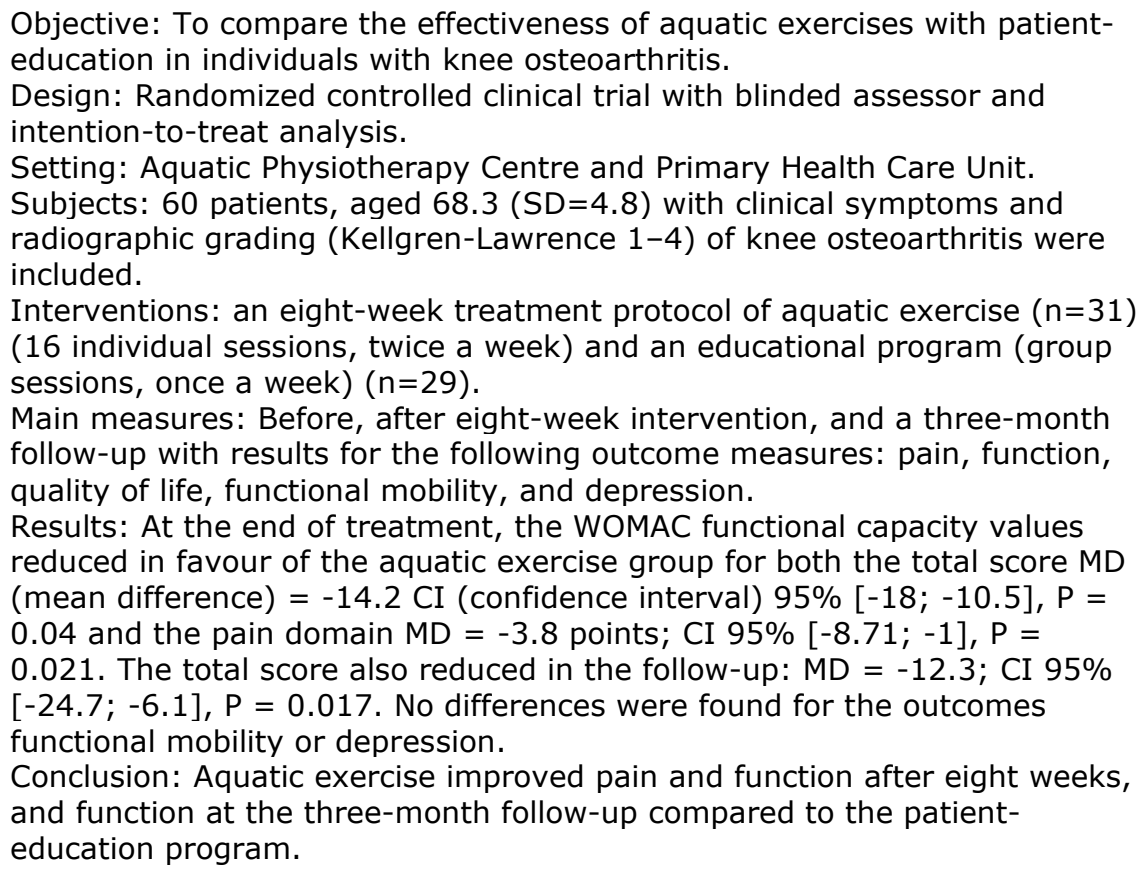 \\
\hline
\end{tabular}

\section{SCHOLARONE \\ Manuscripts}


1 Effectiveness of aquatic exercises compared to patient-education on

2 health status in individuals with knee osteoarthritis: A randomized

3 controlled trial.

4

5 Introduction

6

Osteoarthritis is known as a degenerative disorder of the joint cartilage

8 associated with hypertrophic bone changes and is the most common form of

9 arthritis, affecting more than 27 million people in the United States. ${ }^{1}$ A variety of

10 factors, including demographic, clinical, and biomechanical aspects have been

11 studied and associated with functional and pain status. ${ }^{2}$ In addition, growing

12 evidence suggests that psychological factors such as anxiety, fear, and

13 depression may also relate to physical function in patients with knee

14 osteoarthritis. $^{3}$

15 There is broad agreement on recommendations from the various

organizations for non-pharmacologic modalities of treatment for knee

17 osteoarthritis such as aerobic, aquatic, and/or resistance exercises,

18 education/self-management, walking, as well as weight loss in overweight

19 patients. ${ }^{4}$ Results of systematic reviews/guidelines have pointed out that

20 physical exercise is the most recommended non-pharmacological intervention

21 for osteoarthritis patients and can reduce pain and enhance physical function of

22 joints affected by osteoarthritis. ${ }^{5,6}$ Evidence with low to moderate quality has

23 demonstrated no important differences in self-management, pain, symptoms,

24 function or quality of life for these patients when compared to self-management 
programs and other interventions such as exercise, social support or acupuncture. ${ }^{7}$ It has not been compared to an aquatic exercise program.

The most recent review about the use of aquatic exercise for the management of knee and hip osteoarthritis showed it can be effective at the end of treatment with a small effect on pain, function, and quality of life. For only knee osteoarthritis, no positive results were found. Moreover, the authors recommended that future studies should be joint-specific and set exercise programs with clearly described type and dose (intensity, frequency, and duration $)^{8}$, besides the comparison among several modalities used by physiotherapy.

Considering the rationale above, the role of self-management programs compared to aquatic exercise still has not been investigated, including a well described joint-specific exercise program and its results in long-term follow-up. Moreover, the aspects of psychosocial outcomes should also be compared for these modalities. Then the aim of this study was to investigate the effectiveness of an aquatic exercise program compared to patient-education for individuals with knee osteoarthritis on pain, function, quality of life, and depression.

\section{Method}

\section{Study Design and Selection Criteria}

A randomized controlled trial lasting 8-weeks, with a three-month followup, according to the Consort-Statement ${ }^{9}$, was conducted at an Aquatic Physiotherapy Centre and in a Primary Health Care Unit between January 2015 
50 and April 2016. This study was registered at ClinicalTrials.gov (NCT02247882).

51 All procedures were approved by the University Ethics Committee

$52 \quad(\# 27913514.8 .0000 .5231)$.

53 Participants were recruited from the local Primary Health Care Unit, after

54 being evaluated by a rheumatologist, who confirmed the diagnosis of knee OA

55 according to the American College of Rheumatology ${ }^{10}$ - including the Kellgren-

56 Lawrence radiographic criteria ${ }^{11}$, aged from 60 to 85 years and presented

57 adequate clinical and cognitive conditions for carrying out activities in the pool,

58 confirmed by the Mini-Mental State Examination (24-30 points). ${ }^{12}$ The Kellgren-

59 Lawrence radiographic criteria indicated that most patients (58\%) had a mild

60 degree (grades 1 and 2); while others (42\%) had a severe stage of radiographic

61 abnormalities (grades 3 and 4).

62 The exclusion criteria were: patients undergoing orthopaedic and

63 neurological surgical procedures, those with coronary diseases, cancer, or

64 uncontrolled hypertension, patients unable to walk without aid equipment,

65 patients with contraindications to practice exercises or enter the pool, those

66 participating in nutrition or physical activity programs in the previous two

67 months, individuals with morbid obesity (body mass index $>40 \mathrm{~kg} / \mathrm{m}^{2}$ ), and

68 those unable to continue the study due to change of address or scheduled

69 hospitalization.

71 Procedures

72

In relation to random allocation process, numbers were generated from

74 the site www.random.org using a random sequence from 1 to 100 , with two 
75

76

77

78

79

80

columns. For allocation concealment, the numbers generated were placed in

sealed, opaque envelopes containing the previously decided group names

Aquatic Exercise or Education Program. The envelopes were numbered and placed in sequence. One individual, not involved with the study, was responsible for the randomization and opening the envelope. After the baseline assessment, this individual informed the participants to which he/she was allocated, either the aquatic exercise or the educational program group.

The participants who met the eligibility criteria were assessed on three different occasions: at baseline, at the end of the treatment (8-weeks), and after a three-month follow-up. Participants were evaluated by two individuals in the morning period at the Laboratory of Biomechanics and Clinical Epidemiology. At the baseline assessment, the participants were informed about all the procedures and possible risks, signed the approved consent form, and anthropometric data (mass and height) were collected. Following these initial procedures, the questionnaires were completed, and the functions test performed.

\section{Study Interventions}

The patient-education group program (five individuals per group) was designed and delivered by a multidisciplinary team: physician, pharmacist, nurse, nutritionist, psychologist, physiotherapist, and physical educator. The classes were weekly (total of eight), lasting two hours and were given at the Primary Health Care Unit. Following the suggestions by Coleman et al., ${ }^{13}$ the guidance on the disease and its complications were included; strategies for pain control (cognitive and pharmacological), physical exercise, nutrition, and weight 
100 control, medications (type, interactions, side effects and updates), balance,

101 proprioception, preventing falls, and how to deal with chronic pain. This group

102 also received home knee osteoarthritis exercise guidelines for practice two to

103 three times a week, which included: warm-up, self-stretching, isometric and

104 dynamic exercises, proprioceptive and functional exercises of the lower limbs,

105 and cool down.

$106 \quad$ The aquatic program was performed individually twice a week, for 8-

107 weeks, each session lasting 60 minutes, totalling 16 sessions, provided by

108 certified physiotherapists in the Aquatic Physiotherapy Centre. The water

109 temperature was maintained at approximately $32{ }^{\circ} \mathrm{C}\left(89^{\circ} \mathrm{F}\right)$, with a depth of

$1101.2 \mathrm{~m}$. The exercise protocol consisted of specific exercises: five minutes of

111 warm-up with walking, patellar mobilization; stretching the leg muscles

112 (quadriceps, gluteus, adductors and abductors of hip, triceps surae, and

113 hamstrings); 15 minutes of knee and hip isometric and dynamic exercises with

114 elastic bands (gluteus, adductors and abductors, quadriceps, hamstrings, and

115 triceps surae); 20 minutes of aerobic exercises (stationary running or deep

116 water-running); 10 minutes of step training and proprioceptive exercises; and 10

117 minutes of cool down with massage and relaxation (Appendix 1). The selected

118 exercises were based on studies for outcomes function, ${ }^{8,14}$ pain, ${ }^{8,14}$ balance, ${ }^{15,16}$

119 and aerobic capacity. ${ }^{17}$

120

121 Study Outcomes

122 The primary outcome measures were pain, assessed by a visual

123 analogue scale ${ }^{18}$ and functional capacity through the Western Ontario \& 
124 McMaster Universities Osteoarthritis Index: WOMAC. ${ }^{19}$ The Minimal Clinically

125 Important Difference for knee OA is -7.9 points for WOMAC total score. ${ }^{20}$

126 As secondary outcomes, quality of life, screen on depression, and

127 functional mobility were recorded. Quality of life was measured using the

128 Medical Outcome Study Short Form 36-item Health Survey (version 2.0) and an

129 improvement of 5 points in the physical component score of the questionnaire is

130 considered to be clinically significant. ${ }^{21}$ The presence of depressive symptoms

131 was defined as obtaining six or more points in the short version (15 items) of the

132 Yesavage Geriatric Depression Scale. ${ }^{22}$ The Timed Up and GO test is a

133 performance-based measure and the minimal detectable change of the test in

134 individuals with grade $1-3$ (Kellgren-Lawrence criteria) for knee osteoarthritis

135 is 1.14 seconds. ${ }^{23}$ The team involved in the study was blinded to which study

136 group the patient belonged to throughout the measurements. Two researchers

137 were involved in the assessment.

Statistical Analysis

140 The sample size was calculated for the outcome of pain using the

141 formula proposed by Pocock ${ }^{24}$ which considered an alpha $=0.05$ and $80 \%$

142 power to detect a reduction of $30 \%$ in pain. ${ }^{8}$ The estimated sample was 60

143 patients in the Aquatic Exercise Group and Educational Program groups.

144 The variables were analysed for normal distribution using the Shapiro-

145 Wilk test and as the normality assumption was accepted, data are presented as

146 mean and standard deviation (SD), mean differences (MD), and 95\%

147 confidence intervals (Cl). A Generalized Estimating Equation ${ }^{25}$ model through a

148 specific syntax was employed for comparison within/between groups. A working 
149 correlation matrix was specified a priori and defined the hypothesized

150 relationship between repeated observations on a subject. The model type was

151 set up as a linear scale response. The standard error estimates were adjusted

152 according to the hypothesized correlation between different time points of the

153 outcome (primary and secondary). Bonferroni tests for analysis by multiple

154 comparisons were applied when appropriate. The statistical significance

155 adopted for all tests was 5\% and performed according to intention-to-treat

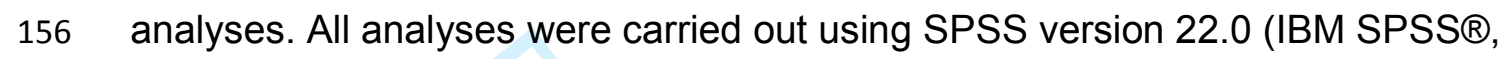

157 Armonk, NY, USA).

158

159

Results

160 eligibility criteria and were randomized between January 2015 and April 2016.

163 Thirty-one were allocated to the Aquatic Exercise group and 29 to the

164 Educational Program group and received the interventions. Two patients were 165 lost due to health problems (pneumonia and panic syndrome) before the follow166 up evaluation, and nine dropped out of the sessions, giving a total of 28 patients 167 in the Aquatic Exercise group and 21 patients in the Educational Program group 168 (dropout rate 18.3\%) (Fig. 1) for follow-up evaluation. No side effects were 169 reported during the treatment in either group.

170 Both groups were similar in the assessed characteristics and outcomes 171 at baseline (Tables 1, 2, and 3). A statistically significant difference was found 172 between groups for the Yesavage questionnaire $P=0.013 ; \mathrm{MD}=-1.795 \% \mathrm{Cl}[-$ 
$1733.76 ;-0.31]$ at baseline, although the scores did not indicate the presence of 174 depression.

175 For the primary outcome of pain, no statistical differences were found 176 between or within groups when assessed by the Visual Analog Scale, but when

177 the pain domain of the WOMAC questionnaire was assessed, statistical

178 changes were found within and between groups in favour of the Aquatic

179 Exercise group. In this group, the pain decreased at the end of treatment MD = $180-3.3$ points; $95 \% \mathrm{Cl}[-6.56 ;-0.1] P=0.031$; and at the follow-up period $\mathrm{MD}=$ $181-3.1$ points; $95 \% \mathrm{Cl}[-6.3 ;-0.03] P=0.046$. At the end of treatment, a significant 182 reduction was noted in favour of the Aquatic Exercise group when compared to 183 the Educational Program group, $\mathrm{MD}=-3.8$ points $95 \% \mathrm{Cl}[-8.7 ;-1] P=0.021$. 184 When function was analysed, WOMAC scores reduced after treatment $185 \mathrm{MD}=-11$ points $95 \% \mathrm{Cl}[-14.9 ;-9.6], P=0.009$ and at the end of follow-up MD $186=-11.8$ points; $95 \% \mathrm{Cl}[-19.3 ;-3.6] ; P=0.020$ compared to baseline in the 187 Aquatic Exercise group. The Minimal Clinically Important Difference was 188 achieved, with $13(41.9 \%)$ individuals overcoming these values at the end of 189 treatment and $14(45.2 \%)$ at the end of the follow-up period. Moreover, the 190 scores statistically reduced in favour of the Aquatic Exercise group both after 191 treatment $\mathrm{MD}=-14.2$ points $95 \% \mathrm{Cl}[-18 ;-10.5], P=0.04$, and at follow-up MD $192=-12.3$ points; $95 \% \mathrm{Cl}[-24.7 ;-6.1], P=0.017$. When comparing the values of 193 Minimal Clinically Important Difference between the groups, the aquatic group 194 achieved improvement at the end of treatment in $13(41.9 \%)$ versus $7(24.1 \%)$ 195 individuals of the Educational Program group, and at the end of follow-up in 14 $196(45.2 \%)$ individuals versus 8 (27.5\%) of the Educational Program group. 
Regarding the secondary outcome quality of life, improvements over time

198

199

200

201

202

203

204

205

206

207

208

209

210

211

212

213

214

215

216

217

218

219

220 in the Aquatic Exercise group were observed, with statistically significant differences at the end of treatment $\mathrm{MD}=9.695 \% \mathrm{Cl}[2.9 ; 16.3], P<0.001$, and at the follow-up period $\mathrm{MD}=10.6 ; 95 \% \mathrm{Cl}[3.5 ; 17.8], P<0.001$. When comparing the Minimal Clinically Important Difference values between the groups, the Aquatic Exercise group achieved improvement in 19 (61.3\%) patients versus $12(41.4 \%)$ individuals in the Educational Program group at the end of treatment, and in $19(61.3 \%)$ versus $16(55.2 \%)$ at the end of follow-up. Functional mobility assessed by the Timed Up and Go test showed no statistically significant differences within/between groups, but the minimum values of detectable change were reached at the end of treatment ( 2.3 seconds; 10 subjects, $34.5 \%)$ and at the end of follow-up (1.3 seconds; 13 subjects, 44.8\%) in the Educational Program group. The depressive symptoms demonstrated no statistically significant differences either within or between, at the end of treatment and follow-up.

\section{Discussion}

This study showed that aquatic exercises, when compared to patienteducation, were superior in improving function and pain in individuals with knee osteoarthritis, while quality of life and depressive symptoms presented no differences. The results of the group submitted to aquatic exercises were effective in improving pain, function, and quality of life after treatment, and function at the end of the three-month follow-up period. 
The results indicated no differences between the groups or within the

222 groups for pain when assessed by the Visual Analog Scale. However, it should

223 be noted that the mean baseline pain was moderate: $4.1 \mathrm{~cm}$ for the aquatic

224 exercises patients and $4.6 \mathrm{~cm}$ for the Educational Program group; there was a

225 decrease for the Aquatic Exercise group of $1.2 \mathrm{~cm}$ at the end of the 8-weeks

226 and in the follow-up this value was maintained. On the other hand, for the

227 Educational Program group, the reduction was 0.8 and $0.9 \mathrm{~cm}$ at the end and

228 follow-up assessments respectively. It is known that the minimal clinically

229 important difference was not established for Visual Analog Scale on

230 osteoarthritis population, however, according to Tubach et al. ${ }^{20}$ the minimal

231 clinically important improvement varies depending on the baseline state:

232 patients who have the most severe symptoms (which represented $48 \%$ of

233 individuals according to the Kellgren-Lawrence criteria) must experience a

234 greater change to consider them improved. In this case, improvements in pain

235 in the present study can be considered satisfactory and must not be discarded

236 within the groups.

237 However, when assessed by the WOMAC questionnaire (pain domain),

238 changes were observed over time for the Aquatic Exercise group and by the

239 end of the treatments between groups, also in favour of the Aquatic Exercise

240 group. It is generally accepted that the WOMAC questionnaire has greater

241 specificity and consequently better responsiveness for people with osteoarthritis

242 when compared to Visual Analog Scale, explaining the improvement just in the

243 WOMAC questionnaire. ${ }^{26}$

$244 \quad$ Aquatic exercise may have effects on pain because of fluid mechanics.

245 The effect of buoyancy could reduce pain during exercise as the depth of 
246 immersion is directly related to the percentage weight bearing. ${ }^{8,27-30}$ The

247 hydrostatic pressure acts compressing the tissues and, in combination with the

248 circulatory changes that occur with immersion, reduces swelling, permitting

249 greater movement to reduce joint and soft-tissue stiffness and, therefore,

250 improve pain complaints. ${ }^{29-30}$

251 A meta-analysis of trials investigating water-based, aerobic and

252 strengthening exercises, and spa therapy for osteoarthritis concluded that all

253 have a positive effect on pain. ${ }^{31}$ A Cochrane review of aquatic therapy for

254 osteoarthritis of the hip or knee also concluded that pain may be decreased by

255 aquatic exercises. ${ }^{8}$ A recent clinical trial investigating aquarobic therapy

256 (several types of exercises including aerobics in water, three times a week in 1-

257 hour sessions, for a total of 36 sessions over 12 weeks) versus patient

258 education (two educational sessions delivered through lectures on osteoarthritis

259 and the necessity of exercising), showed a statistically significant difference in

260 pain. $^{32}$

261 The present study presented some similar methodological elements

262 when compared to the aforementioned studies, for example, time of the

263 sessions with a duration of 60 minutes, a minimum weekly frequency of two

264 times and a minimum duration of eight weeks of intervention. When confronted

265 with the types of exercises used in the programs, the clear majority (and the

266 present study) was composed of warm-up, flexibility, dynamic and aerobic

267 exercises. The present study differed in the addition of balance exercises,

268 proprioceptive, deep-water running in the aerobic component and relaxation

269 with the addition of massage in the periarticular musculature of the knees. 
Educational programs have been statistically proven to be good in reducing pain, as evaluated by Coleman et al., ${ }^{33}$ with a 6 -week knee specific self-management education program, delivered by health professionals. In a recent review including 29 studies (6,753 participants) Kroon et al., ${ }^{7}$ found that educational programs mildly reduced pain when compared with usual care. In the current study, the Aquatic Exercise group improved function over time and presented better results than the Educational Program group. The Aquatic Exercise group values of minimal clinically important difference from the WOMAC questionnaire were achieved at the end of treatment and at the followup period.

This positive result was also reported in the systematic reviews published by Barker et al., ${ }^{34}$ and Bartels et al., ${ }^{8}$ aquatic therapy mildly improved physical function both in patients affected by musculoskeletal, and in patients with combined hip and knee osteoarthritis. In another systematic review, aquatic physiotherapy was compared with exercises on land by Batterham et al. ${ }^{14}$ for function, mobility, and health outcomes. No favourable results were found for either group. In conclusion, the authors suggested the option of aquatic exercises for individuals who have difficulty in attending on land.

Functional improvements were reflected by changes in several measured parameters, such as pain and quality of life. It is generally accepted that the WOMAC questionnaire has greater specificity and consequently better responsiveness for people with osteoarthritis; ${ }^{26}$ nonetheless, the Medical Outcome Study Short Form 36-item Health Survey also reflected these changes. 
294

295

296

297

298

299

300

301

302

303

304

305

306

307

308

309

310

311

312

313

314

315

316

317

318

Educational programs have also proved beneficial in improving function

in some clinical trials: Bezalel et al., ${ }^{35}$ reported a reduction in the WOMAC total score after four weeks of treatment and at the 8-week follow-up, between

education and control groups. Similar findings were listed by Carvalho et al., ${ }^{36}$ after three months of treatment. Coleman et al., ${ }^{33,37}$ reported improvements in WOMAC total score at the end of a 12-month treatment, as well as after an 8week intervention and a 6-month follow-up period. Contrary to these findings, a recent review did not show differences between self-management programs or any other intervention for the function outcome. ${ }^{7}$

In the present study, positive effects were seen in quality of life in the Aquatic Exercise group and the minimal detectable change values were achieved at the end and at follow-up period. Two systematic reviews showed improvement in quality of life using the aquatic therapy modality. Bartels et al.. ${ }^{13}$ at the end of aquatic exercise treatment for combined knee and hip osteoarthritis, showed a small effect on quality of life. Moderate improvements were reached by Barker et al., ${ }^{34}$ when comparing aquatic exercise with no exercise for musculoskeletal conditions.

The reasons that justify the effectiveness of educational programs for health outcomes are still not well understood and can be justified by many different factors. Moreover, the meta-analysis of educational programs has concluded that it is difficult to compare models between different chronic conditions, which is also the case for different types of arthritis. ${ }^{38}$ The present educational program was developed specifically for the population with knee osteoarthritis, aimed at decreasing pain as well as improving function and quality of life, delegated by professionals with 
319

320

321

322

323

324

325

326

327

328

329

330

331

332

333

334

335

336

337

338

339

340

341

342

343

experience. Information about the disease and the benefits of exercise were

incorporated into the constructs of self-knowledge to improve self-efficacy and

changes in the behaviour of these individuals. Using the knowledge and skills of

health professionals is a major component of the educational program because

knowledge is an important part of self-efficacy and no amount of trust will

succeed unless the necessary knowledge and skills are present. ${ }^{39}$

Understanding the rationale for adopting concepts in the program allows

participants to become self-motivated to change behaviour and thus to be more adherent in the long term. ${ }^{40}$

In the present investigation, functional mobility, assessed by the Timed

Up and Go test, did not demonstrate significant differences within or between

groups, but in the Educational Program the minimal detectable change was

achieved at the end of the treatment ( 2.3 seconds) and at the follow-up period

(1.3 seconds). The same test was investigated in a clinical trial which compared

an orientation (manual with guidelines on how not to overload the knee in daily

activities and instructions for pain and medication) and an exercise group (on

land, twice a week, 8 weeks, involving stretching and strengthening of the

quadriceps). ${ }^{41}$ At the end of the treatment there was no statistically significant

difference in the pre-and post-intervention evaluation in the orientation group.

However, in the exercise group, there was a statistically significant

difference in Timed Up and Go test scores. Comparing the groups, a higher

improvement in the Timed Up and Go test in the exercise group compared to

the orientation group was observed. When investigating water based exercise,

a recent study compared the effects of two aquatic exercise programs (aqua-

fitness program and seated aqua-based exercise program) on physical function 
344 for 12 weeks in individuals with osteoarthritis (hip, hands, knee or spine) and no

345 significant changes in Timed Up and Go test were observed. ${ }^{42}$ Both instruments

346 (WOMAC and Timed Up and Go test) were used to evaluate function, but

347 WOMAC showed better improvement compared to Timed Up and Go test,

348 regarding its greater specificity to people with osteoarthritis and consequently

349 better responsiveness.

350 With respect to the screen on depression, no differences were found

351 between or within the groups of the present study. At baseline, the individuals

352 showed no signs or symptoms of depression (<6 points from the questionnaire)

353 and both treatments appeared to maintain this status. Scopaz et al., ${ }^{3}$

354 investigated the association between fear, anxiety, and depression with physical

355 function in individuals with knee osteoarthritis. Depression may influence scores

356 in function under conditions of low anxiety and no results were found when

357 correlating the Timed Up and Go test and depression.

358 Axford et al. ${ }^{43}$ proposed a clinical trial (educational versus no treatment)

359 consisting of four 1 -h group sessions led by a trained registered nurse. The

360 sessions covered information about the disease, medication and other

361 treatments, activities (exercise and relaxation), and skills (strategies for pain

362 management) guided by a special booklet for both groups. A complex

363 interrelationship between depression, pain, disease knowledge, and physical

364 ability in patients with knee osteoarthritis was demonstrated. All patients

365 showed a progressive decrease in mental health over the duration of the study

366 and greater pain scores were associated with reduced coping, increased

367 depression, and reduced physical ability. The authors concluded that the

368 treatment of depression and pain may be paramount to the successful 
369 treatment of knee osteoarthritis, and these factors should be considered for

370 each patient. Kim et al., ${ }^{44}$ investigated depression symptoms with another

371 questionnaire comparing a non-equivalent control group and 36 sessions of an

372 aquarobic exercise program (60-minute sessions, three times per week). At the

373 end of the protocol, the aquarobic group presented significantly reduced

374 depression values compared to the control group.

375 Some limitations of this study are listed as follows: a high dropout rate

376 (especially in the Educational Program group) may have jeopardized the

377 results, even using the intention-to-treat analysis. The Education Program

378 Group does not receive an equivalent amount of supervised land-based

379 exercise when compared to the Aquatic Exercise group. The heterogeneity of

380 the groups in relation to the outcome of depressive symptoms in the baseline

381 evaluation should also be taken into consideration.

382 New clinical trials are needed to confirm the effects of aquatic exercise

383 and educational programs on patients with knee osteoarthritis, including the

384 cost-effectiveness outcome. High quality studies that follow the

385 recommendations of the Consort-Statement ${ }^{20}$ are required, as well as

386 standardization of outcomes and interventions to facilitate comparisons. 


\section{Clinical Messages}

395

396

Aquatic exercise program (16 sessions, twice a week) was superior when

397

compared to the educational program (eight sessions, weekly, lasting two

398

hours) in pain and function, at the end eight weeks and after three-month follow-

399

up, for patients with knee osteoarthritis.

400

401

\section{Conflict of interest}

402

403

The authors declared no potential conflicts of interest with respect to the

404

research, authorship, and/or publication of this article.

405

406

407

\section{References}

408

409

410

411

1. Lawrence RC, Felson DT, Helmick CG, et al. Estimates of the prevalence of arthritis and other rheumatic conditions in the United States. Arthritis Rheum

2. Fitzgerald GK, Piva SR, Irrgang JJ, Bouzubar F and Starz TW. Quadriceps 2008; 58: 26-35. activation failure as a moderator of the relationship between quadriceps strength and physical function in individuals with knee osteoarthritis. Arthritis 
419 3. Scopaz KA, Piva SR, Wisniewski S and Fitzgerald GK. Relationships of fear,

420 anxiety, and depression with physical function in patients with knee

421 osteoarthritis. Arch Phys Med Rehabil 2009; 90: 1866-1873.

422

423

4. Hochberg MC, Altman RD, April KT, et al. American College of

Rheumatology 2012 recommendations for the use of nonpharmacologic and pharmacologic therapies in osteoarthritis of the hand, hip, and knee. Arthritis Care Res 2012; 64: 465-474.

427

5. McAlindon TE, Bannuru RR, Sullivan MC, Arden NK, Berenbaum F and Bierma-Zeinstra SM, et al. OARSI guidelines for the non-surgical management of knee osteoarthritis. Osteoarthr Cartil 2014; 22: 363-388.

6. Fransen M, McConnell S, Harmer AR, Van der Esch M, Simic M and Bennell KL. Exercise for osteoarthritis of the knee. Cochrane Database Syst Rev 2015; 1: CD004376.

435

7. Kroon FPB, Van der Burg LRA, Buchbinder R, Osborne RH, Johnston RV and Pitt V. Self-management education programmes for osteoarthritis. Cochrane Database Syst Rev 2014; 1: CD008963. 
444 9. Schulz KF, Altman DG, Moher D and CONSORT Group. CONSORT 2010

445 statement: updated guidelines for reporting parallel group randomised trials.

$446 \quad$ Int J Surg 2011; 9: 672-677.

447

448

449

10. American College of Rheumatology Subcommittee on Osteoarthritis. Recommendations for the medical management of osteoarthritis of the hip

12. Lancu I and OImer A. The mini-mental state examination--an up-to-date review. Harefuah 2006; 145: 687-690.

13. Coleman S, McQuade J, Rose J, Inderjeeth C, Carroll G and Briffa NK. Selfmanagement for osteoarthritis of the knee: Does mode of delivery influence outcome? BMC Musculoskelet Disord 2010; 11: 56.

14. Batterham SI, Heywood S, Keating JL. Systematic review and meta-analysis comparing land and aquatic exercise for people with hip or knee arthritis on function, mobility and other health outcomes. BMC Musculoskelet Disord $2011 ; 12: 123$. 
469 15. Lund H, Weile U, Christensen R, Rostock B, Downey A, Bartels EM, et al. A

470 randomized controlled trial of aquatic and land-based exercise in patients

471 with knee osteoarthritis. J Rehabil Med 2008; 40: 137-144.

472

473

16. Hale LA, Waters D and Herbison P. A randomized controlled trial to

474 investigate the effects of water-based exercise to improve falls risk and

475 physical function in older adults with lower-extremity osteoarthritis. Arch Phys Med Rehabil 2012; 93: 27-34.

477

17. Escalante Y, García-Hermoso A and Saavedra JM. Effects of exercise on functional aerobic capacity in lower limb osteoarthritis: A systematic review. J Sci Med Sport 2011; 11: 190-198.

18. Price DD, Rafii A and Buckingham $B$. The validation of visual analogue scales ratio scale measures for chronic and experimental pain. Pain 1983; 17: 45-56.

485

19. Bellamy N, Buchanan WW, Goldsmith CH, Campbell J and Stitt LW. Validation study of WOMAC: a health status instrument for measuring clinically important patient relevant outcomes to antirheumatic drug therapy in patients with osteoarthritis of the hip or knee. J Rheumatol 1988; 15: 1833-1840.

491

492 
493 20. Tubach F, Ravaud P, Baron G, Falissard B, Logeart I, Bellamy N, et al.

494 Evaluation of clinically relevant changes in patient reported outcomes in

495 knee and hip osteoarthritis: the minimal clinically important improvement.

496 Ann Rheum Dis 2005; 64: 29-33.

497

498

499

21. Ware JE Jr, Kosinski MA. SF-36 Physical \& mental health summary Scales:

$500 \quad$ Quality Metric Inc., 2002.

501

502

503

504

505

506

507

508

509

510

25. Hanley JA, Negassa A, Edwardes MDB and Forrester JE. Statistical analysis of correlated data using generalized estimating equations: an 516

23. Alghadir A, Anwer S and Brismée JM. The reliability and minimal detectable change of Timed Up and Go test in individuals with grade 1-3 knee osteoarthritis. BMC Musculoskelet Disord 2015; 16: 174.

24. Pocock SJ. Clinical Trials: a practical approach. Chichester: John Wiley \& Sons, 1983. orientation. Am J Epidemiol 2003; 157: 364-375. 
517 26. Davies M, Watson DJ and Bellamy N. Comparison of the responsiveness

518 and relative effect size of the Western Ontario and McMaster Universities

519 Osteoarthritis Index and the Short-Form Medical Outcomes Study Survey in

520 a randomized, clinical trial of osteoarthritis patients. Arthritis Care Res

$521 \quad 1999,12: 172-179$.

522

523 27. Verhagen AP, Cardoso JR and Bierma-Zeinstra SM. Aquatic exercise \&

524 balneotherapy in musculoskeletal conditions. Best Pract Res Clin Rheumatol

$525 \quad 2012 ; 26: 335-343$.

526

527 28. Harrison RA, Hillman M, Bustrode S. Loading of the lower limb when 528 walking partially immersed: implications for clinical practice. Physiotherapy 529 1992; 78: 164-166.

530

29. Lin S-C, Davey R and Cochrane T. Community rehabilitation for older adults with osteoarthritis of the lower limb: A controlled clinical trial. Clin Rehabil

534

30. Hinman RS, Heywood SE and Day AR. Aquatic physiotherapy for hip and knee osteoarthritis: results of a single-blind randomised controlled trial. Phys Ther 2007; 87: 32-43. 
541 31. Zhang W, Moskowitz RW, Nuki G, Abramson S, Altman RD, Arden N, et al.

542 OARSI recommendations for the management of hip and knee

543 osteoarthritis, part 1: critical appraisal of existing treatment guidelines and

544 systematic review of current research evidence. Osteoarthr Cartil 2007; 15:

$545981-1000$.

546

547

548

549

550

551

552

553

554

555

556

557

558

559

560

561

562

563

564

565

33. Coleman S, Briffa K, Conroy H, Prince R, Carroll G and McQuade J. Short and medium-term effects of an education self-management program for individuals with osteoarthritis of the knee, designed and delivered by health professionals: a quality assurance study. BMC Musculoskelet Disord 2008; 9: 117 .

34. Barker AL, Talevski J, Morello RT, Brand CA, Rahmann AE and Urquhart DM. Effectiveness of aquatic exercise for musculoskeletal conditions: a meta-analysis. Arch Phys Med Rehabil 2014; 95: 1776-86.

35. Bezalel T, Carmeli E and Katz-Leurer M. The effect of a group education programme on pain and function through knowledge acquisition and homebased exercise among patients with knee osteoarthritis: A parallel randomised single-blind clinical trial. Physiotherapy 2010; 96: 137-143. 
566 36. Carvalho NA, Bittar ST, Pinto FR, Ferreira M and Sitta RR. Manual for

567 guided home exercises for osteoarthritis of the knee. Clinics (Sao Paulo)

$568 \quad 2010 ; 65: 775-780$.

569

570

571

572

573

37. Coleman S, Briffa NK, Carroll G, Inderjeeth C, Cook N and McQuade J. A randomised controlled trial of a self-management education program for osteoarthritis of the knee delivered by health care professionals. Arthritis Res Ther 2012; 14: R21.

574

575

576

577

578

579

580

581

582

583

584

39. Pajares F: Overview of social cognitive theory and self-efficacy Atlanta:

38. Warsi A, Wang PS, LaValley MP, Avorn J, Solomon DH. Self-management education programs in chronic disease. A systematic review and methodological critique of the literature. Arch Intern Med 2004; 164: 1641 1649.

586

587

588

589

40. Elder J, Ayala G, Harris S: Theories and intervention approaches to healthbehaviour change in primary care. Am J Prev Med 1999, 17: 275-284.

41. Oliveira AMI, Peccin MS, Kelson Silva KNG, Teixeira LEPP and Trevisani VFM. Impacto dos exercícios na capacidade funcional e dor em pacientes com osteoartrite de joelhos: ensaio clínico randomizado. Rev Bras Reumatol 590 2012; 52: 870-882. 
591

592 42. Fisken AL, Waters DL, Hing WA, Steele M and Keogh JW. Comparative

593 effects of 2 aqua exercise programs on physical function, balance, and

594 perceived quality of life in older adults with osteoarthritis. J Geriatr Phys

$595 \quad$ Ther 2015; 38: 17-27.

596

597 43. Axford J, Heron C, Ross F and Victor CR. Management of knee

598 osteoarthritis in primary care: Pain and depression are the major obstacles.

$599 \quad J$ J Psychosom Res 2008; 64: 461-467.

600

601 44. Kim IS, Chung SH, Park YJ and Kang HY. The effectiveness of an

602 aquarobic exercise program for patients with osteoarthritis. Appl Nurs Res

$603 \quad 2012 ; 25: 181-189$. 


\section{ABSTRACT}

2 Objective: To compare the effectiveness of aquatic exercises with patient-

3 education in individuals with knee osteoarthritis.

4 Design: Randomized controlled clinical trial with blinded assessor and

5 intention-to-treat analysis.

6 Setting: Aquatic Physiotherapy Centre and Primary Health Care Unit.

7 Subjects: 60 patients, aged $68.3(\mathrm{SD}=4.8)$ with clinical symptoms and

8 radiographic grading (Kellgren-Lawrence 1-4) of knee osteoarthritis were

9 included.

10 Interventions: an eight-week treatment protocol of aquatic exercise $(n=31)$ (16

11 individual sessions, twice a week) and an educational program (group sessions,

12 once a week) $(n=29)$.

13 Main measures: Before, after eight-week intervention, and a three-month

14 follow-up with results for the following outcome measures: pain, function, quality

15 of life, functional mobility, and depression.

16 Results: At the end of treatment, the WOMAC functional capacity values

17 reduced in favour of the aquatic exercise group for both the total score MD

18 (mean difference) $=-14.2 \mathrm{Cl}$ (confidence interval) 95\% [-18; -10.5], $P=0.04$

19 and the pain domain $\mathrm{MD}=-3.8$ points; $\mathrm{Cl} 95 \%[-8.71 ;-1], P=0.021$. The total

20 score also reduced in the follow-up: $\mathrm{MD}=-12.3 ; \mathrm{Cl} 95 \%[-24.7 ;-6.1], P=0.017$.

21 No differences were found for the outcomes functional mobility or depression.

22 Conclusion: Aquatic exercise improved pain and function after eight weeks,

23 and function at the three-month follow-up compared to the patient-education

24 program.

25 Keywords: self-care, hydrotherapy, knee osteoarthritis, pain, depression, 
Table 1. Baseline characteristics of participants.

\begin{tabular}{|c|c|c|c|}
\hline & $A E(n=31)$ & $\operatorname{EP}(n=29)$ & \\
\hline \multicolumn{4}{|l|}{ Gender } \\
\hline Male n (\%) & $8(25.8)$ & $11(37.9)$ & $P=0,37$ \\
\hline Female $\mathrm{n}(\%)$ & $23(74.2)$ & $18(62.1)$ & $P=0,16$ \\
\hline Age (years) & $67.3(5.9)$ & $68.7(6.7)$ & $P=0,21$ \\
\hline $\mathrm{BMI}\left(\mathrm{kg} / \mathrm{m}^{2}\right)$ & $29.2(0.8)$ & $30.4(0.9)$ & $P=0,42$ \\
\hline
\end{tabular}


Table 2. Summary of the primary outcome measures.

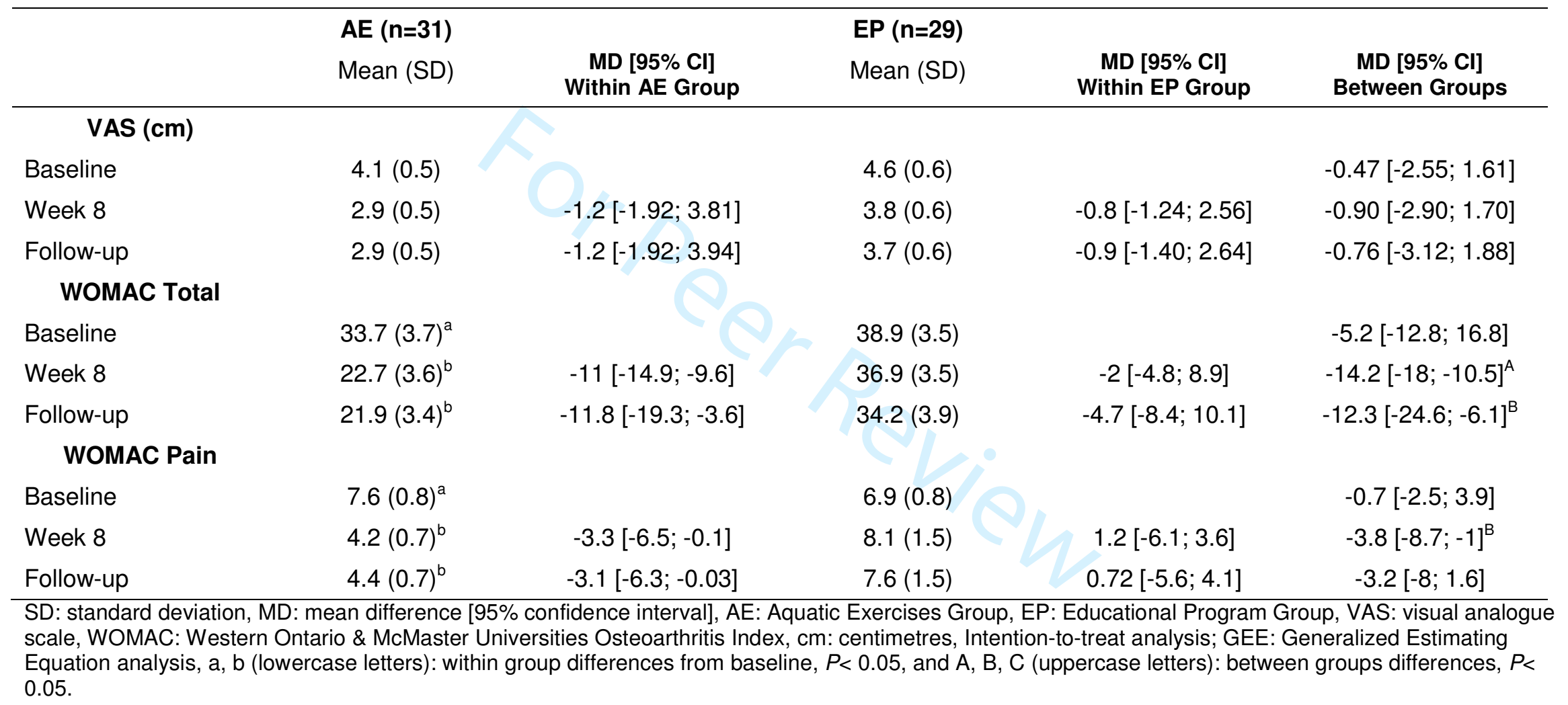


Table 3. Summary of the secondary outcome measures.

\begin{tabular}{|c|c|c|c|c|c|}
\hline & $\operatorname{AE}(n=31)$ & & EP $(n=29)$ & & \\
\hline & Mean (SD) & $\begin{array}{c}\text { MD }[95 \% \text { Cl] } \\
\text { Within AE Group }\end{array}$ & Mean (SD) & $\begin{array}{c}\text { MD [95\% Cl] } \\
\text { Within EP Group }\end{array}$ & $\begin{array}{c}\text { MD }[95 \% \mathrm{Cl}] \\
\text { Between Groups }\end{array}$ \\
\hline \multicolumn{6}{|c|}{ SF-36 (Physical Function) } \\
\hline Baseline & $64.7(3.1)^{\mathrm{a}}$ & & $57.4(3.1)$ & & $7.3[-5.5 ; 20.2]$ \\
\hline Week 8 & $74.3(2.9)^{b}$ & $9.6[2.9 ; 16.3]$ & $61.5(4.1)$ & $4.1[-13.5 ; 5.2]$ & $12.8[-1.7 ; 27.4]$ \\
\hline Follow-up & $75.4(3)^{\mathrm{b}}$ & $10.6[3.6 ; 17.8]$ & $61(4.1)$ & $3.6[-12.7 ; 5.4]$ & $14.3[-0.3 ; 29.1]$ \\
\hline \multicolumn{6}{|c|}{ Yesavage Scale } \\
\hline Baseline & $2.5(0.4)$ & & $4.2(0.5)$ & & $-1.7[-3.7 ;-0.3]^{A}$ \\
\hline Week 8 & $2.4(0.5)$ & $-0.1[-3.4 ; 1.4]$ & $3.5(0.5)$ & $-0.7[-0.9 ; 2]$ & $-1.06[-3.2 ;-1]$. \\
\hline Follow-up & $2.4(0.5)$ & $-0.1[-3.4 ; 1.4]$ & $3.9(0.6)$ & $-0.3[-1 ; 1.7]$ & $-1.4[-3.7 ; 0.9]$ \\
\hline \multicolumn{6}{|c|}{ TUG (s) } \\
\hline Baseline & $11.2(0.8)$ & & $14.7(2.5)$ & & $-3.5[-3.7 ; 2]$ \\
\hline Week 8 & $11.4(0.7)$ & $0.2[10.2 ; 12.5]$ & $12.4(0.8)$ & $-2.3[-3.3 ; 2]$ & $-0.9[-4.7 ; 0.5]$ \\
\hline Follow-up & $11.6(0.7)$ & $0.4[10.2 ; 12.8]$ & $13.4(1.1)$ & $-1.3[-4.3 ; 1.3]$ & $-2[-5.9 ; 1.7]$ \\
\hline
\end{tabular}


Figure 1. Flow Diagram.

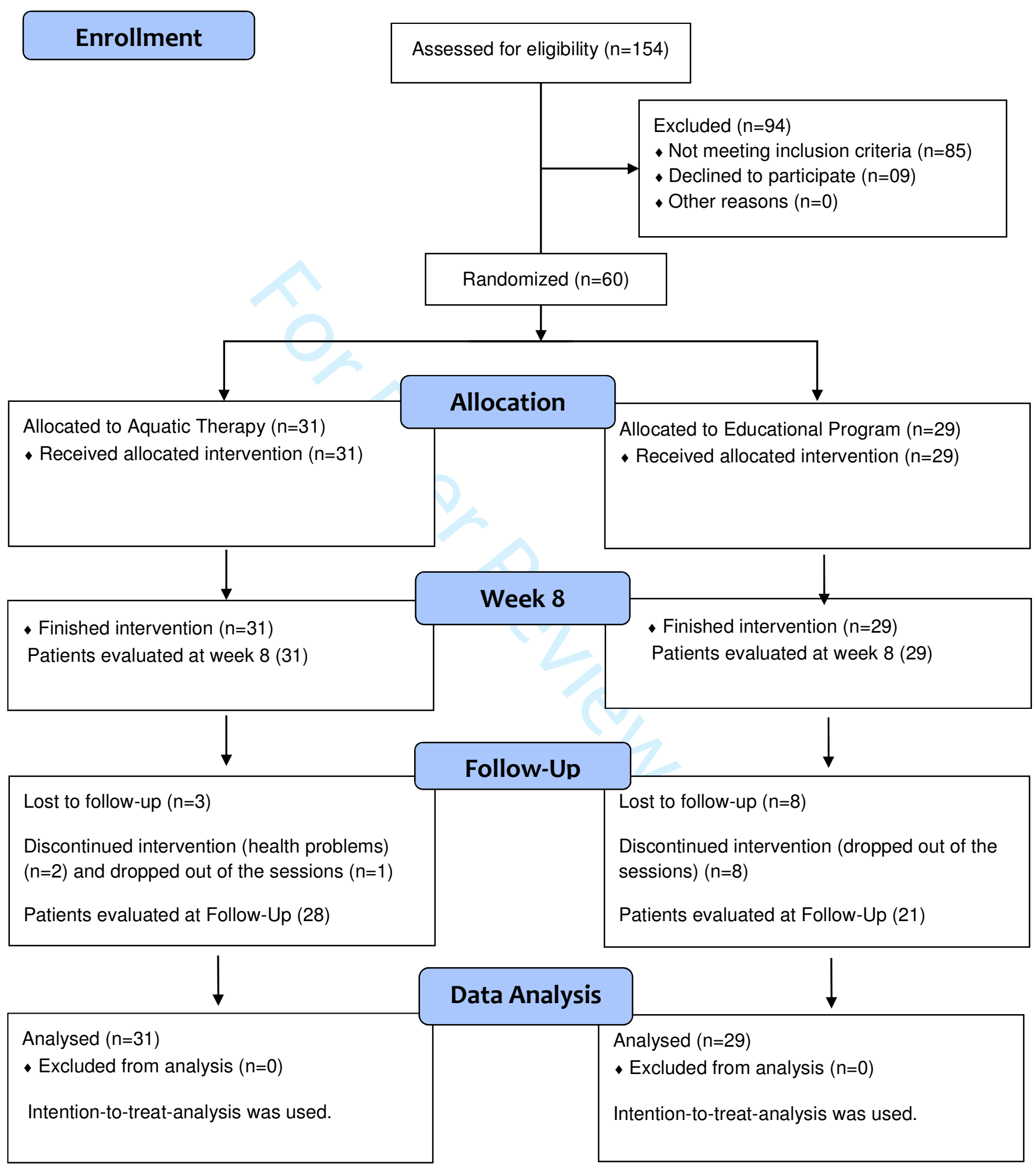


Appendix 1. Aquatic exercises protocol.

\begin{tabular}{|c|c|}
\hline $1^{\text {st }}$ and $2^{\text {nd }}$ weeks & 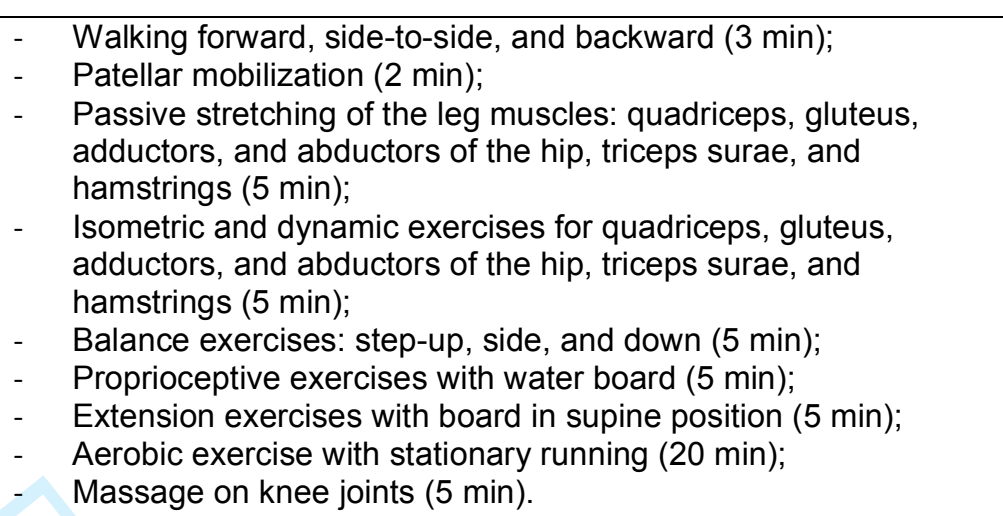 \\
\hline $3^{\text {rd }}$ and $4^{\text {th }}$ weeks & $\begin{array}{l}\text { Walking forward, side-to-side, and backward with elastic band } \\
\text { ( } 3 \text { min); } \\
\text { Patellar mobilization ( } 2 \text { min); } \\
\text { Active stretching of the leg muscles: quadriceps, gluteus, } \\
\text { adductors, and abductors of the hip, triceps surae, and } \\
\text { hamstrings ( } 5 \text { min); } \\
\text { Isometric and dynamic exercises with elastic band for } \\
\text { quadriceps, gluteus, adductors, and abductors of the hip, } \\
\text { triceps surae, and hamstrings ( } 5 \text { min); } \\
\text { Balance exercises: step-up, side, and down with elastic band (5 } \\
\text { min); } \\
\text { Proprioceptive exercises with water board with eyes closed (5 } \\
\text { min); } \\
\text { Extension exercises with board in prone position - swimming } \\
\text { leg (5 min); } \\
\text { Aerobic exercise with aquatic bike (20 min); } \\
\text { Relaxation in supine position (5 min). }\end{array}$ \\
\hline $5^{\text {th }}$ to $8^{\text {th }}$ weeks & $\begin{array}{l}\text { Walking forward, side-to-side, and backward with elastic band } \\
\text { ( } 3 \text { min); } \\
\text { - Patellar mobilization ( } 2 \text { min); } \\
\text { - Active stretching of the leg muscles: quadriceps, gluteus, } \\
\text { adductors, and abductors of the hip, triceps surae, and } \\
\text { hamstrings ( } 5 \text { min); } \\
\text { - Isometric and dynamic exercises with elastic band for } \\
\text { quadriceps, gluteus, adductors, and abductors of the hip, } \\
\text { triceps surae, and hamstrings ( } 5 \text { min); } \\
\text { - Balance exercises with step: kicks and squats ( } 5 \mathrm{~min}) ; \\
\text { - } \quad \text { Proprioceptive exercises with spaghetti ( } 5 \text { min); } \\
\text { - Extension exercises with board in supine and prone position (5 } \\
\text { min); } \\
\text { - Aerobic exercise with deep-water running ( } 20 \mathrm{~min}) ; \\
\text { - Massage on quadriceps and triceps surae (5min). }\end{array}$ \\
\hline
\end{tabular}


1 Acknowledgements: The authors thank the employees of the Primary Health

2 Care Unit "Vila Brasil" and the assistant pool manager of the Aquatic

3 Physiotherapy Centre "Paulo A. Seibert", Mr. Anselmo Borges, for their careful

4 and valuable help during the study.

5

6 Funding: This study was supported by the Araucaria Research Foundation

7 PPSUS/SESA-PR/MS-Decit/CNPq (\# 04/2013) and CNPq (National Council for

8 Scientific and Technological Development) grants.

10 Contributors:

11

12 Ligia Facci and Jefferson Cardoso were responsible for conducting the RCT, 13 mentoring the students, and managing the grants. Celita Trelha, Fernanda

14 Melo, and Daniela Silva supervised the Education Program group at the

15 Primary Health Care Unit. Marcelo Taglietti, Geovane Sawczuk, Thamires

16 Ruivo, and Thaisley Souza conducted the physical examination, assessed the

17 outcome measures, and carried out the exercises at the Aquatic Physiotherapy

18 Centre "Paulo A. Seibert". Chiarella Sforza mentored the PhD student Marcelo

19 Taglietti at the Università degli Studi di Milano and, along with Jefferson

20 Cardoso, was responsible for the statistical analysis. 